\title{
La competencia transversal Diseño y Proyecto aplicada a los objetivos de desarrollo sostenible 7 y 10 en trabajos finales de grado y máster codirigidos entre Universidades europeas
}

\author{
Modesto Pérez-Sánchez $^{\mathrm{a}}$, Paulo J. Branco ${ }^{\mathrm{b}}$, João F. Fernandes ${ }^{\mathrm{b}}$, P. Amparo López-Jiménez ${ }^{\mathrm{a}}$ \\ ${ }^{a}$ Departamento de Ingeniería Hidráulica y Medio Ambiente. Universitat Politècnica de València. mopesan1@upv.es, \\ palopez@upv.es; ${ }^{\mathrm{b}}$ Departamento de Engenharia Eletrotécnica y de Computadores. Instituto Superior Técnico.. \\ pbranco@tecnico.ulisboa.pt, joao.f.p.fernandes@tecnico.ulisboa.pt;
}

\begin{abstract}
The current communication shows the experience that is developed between DIHMA-UPV and DIEC-IST professors. This project is focused on the development of bachelor's and master's thesis focused on improving the Sustainable Development Goals in undevoloped areas, particularly in the case of energy access and its generation through renewable hybrid systems. The development of this experience can be carried out thanks to the updating of asynchronous learning applied methodologies. It is since the students come from degrees, which have only acquired basic learning results in hydraulics or electricity. The development of the activity and its coordination between professors from both universities is established through the use of faculty stays at Erasmus + STA.
\end{abstract}

Keywords: Sustainable development Goals; Renewable engineering; Self-learning.

\begin{abstract}
Resumen
La presente comunicación muestra la experiencia que está desarrollándose entre profesores del DIHMA-UPV y del DIEC-IST. Este proyecto está focalizado en el desarrollo de trabajos finales de grado y máster centrados en la mejora de los Objetivos de Desarrollo Sostenible en zonas desfavorecidas, particularizado al caso del acceso de la energía y su generación mediante sistemas híbrido renovables. El desarrollo de esta experiencia puede llevarse a cabo gracias a la incorporación de metodologías de aprendizaje asíncrono ya que los alumnos proceden de titulaciones que solo han adquirido resultados de aprendizaje básicos en hidráulica o electricidad. El desarrollo de la actividad y su coordinación entre profesores de ambas universidades está establecido mediante el uso de las estancias del profesorado en Erasmus + STA.
\end{abstract}

Palabras clave: Objetivo de desarrollo sostenible; Ingeniería renovable; Aprendizaje autónomo.;

\section{Introducción}

Uno de los principales resultados de la Conferencia Río+20 fue el acuerdo para establecer Objetivos de Desarrollo Sostenible (ODS). Los términos más comunes en los 17 objetivos son crecimiento económico, resiliencia e inclusión. Entre los 17 objetivos, el séptimo hace referencia al uso de la energía. Actualmente y en relación con el acceso a la energía eléctrica, es un hecho que no todos los habitantes del planeta pueden recurrir al mismo. Concretamente, según el Banco Mundial, el 12.2\% de la población mundial 
todavía no dispone de acceso a la energía eléctrica, con porcentajes superiores al $90 \%$ si se analizan los países en vías de desarrollo (Banco Mundial, 2019). Entre las metas que se incluyen, el objetivo dedicado a la energía propone (ODS, 2019):

- Garantizar el acceso universal a servicios energéticos asequibles, fiables y modernos.

- Aumentar considerablemente la proporción de energía renovable en el conjunto de fuentes energéticas.

- Duplicar la tasa mundial de mejora de la eficiencia energética.

- Aumentar la cooperación internacional para facilitar el acceso a la investigación y la tecnología relativas a la energía limpia, incluidas las fuentes renovables, la eficiencia energética y las tecnologías avanzadas y menos contaminantes de combustibles fósiles, y promover la inversión en infraestructura energética y tecnologías limpias.

Además, la propuesta de sistemas que permitan una generación de electricidad desconectada de la red, permitirá trabajar parcialmente el décimo ODS, que está focalizado en la reducción de las desigualdades entre países desarrollados y en desarrollo.

Es por ello, que desde la Universidad Politècnica de València, en la misma línea que la mayoría de las organizaciones, han establecido una línea de trabajo en base a los ODS establecidos con el objetivo de que, desde las actividades docentes, de transferencia e investigación puedan abordarse algunas de las 169 metas establecidas para 2030. Aprovechando los trabajos de investigación entre profesores del Departamento de Ingeniería Hidráulica de la Universitat Politècnica de València (DIHMA-UPV) y del Departamento de Ingeniería Electrónica y Computadores del Instituto Superior Tècnico de Lisboa (Portugal) (DIEC-IST), se han desarrollado propuestas de trabajos finales de grado y máster para que los estudiantes puedan llevar a cabo la aplicación de las competencias específicas del título, competencias transversales así como tengan relación con los objetivos de desarrollo sostenible planteados por la Agenda 2030.

En este aspecto, los ODS podrían abordarse de manera crítica en la educación superior combinando enfoques de enseñanza instrumentalista, práctico y con enfoque plural integrador, que busque en el desarrollo del individuo apoyado en el concepto de la sostenibilidad de los recursos que utiliza (Kopnina, 2018). La presente comunicación muestra la experiencia llevada a cabo entre docentes de la UPV y del IST, donde se ha puesto de manifiesto la capacidad de coordinación transversal de competencias específicas y transversales, siendo integrada en metas incluidas de los ODS.

\section{Objetivos}

El objetivo de la presente comunicación es mostrar la capacidad potencial de trabajo que puede desarrollarse como medida colaborativa entre Universidades europeas, que impliquen el desarrollo de trabajos final de grado (TFG) y/o de máster (TFM). Estos TFG y TFM no solo se basan en las competencias específicas que los alumnos han adquirido a lo largo de los estudios de grado o máster, sino que integran habilidades de competencias transversales que los estudiantes han adquirido en su proceso de formación. Del mismo modo, apoyado en los dos anteriores, los TFG y TFM incluyen metodologías desarrolladas o aplicadas a casos de estudio, que puedan integrar los ODS (Figura 1). 


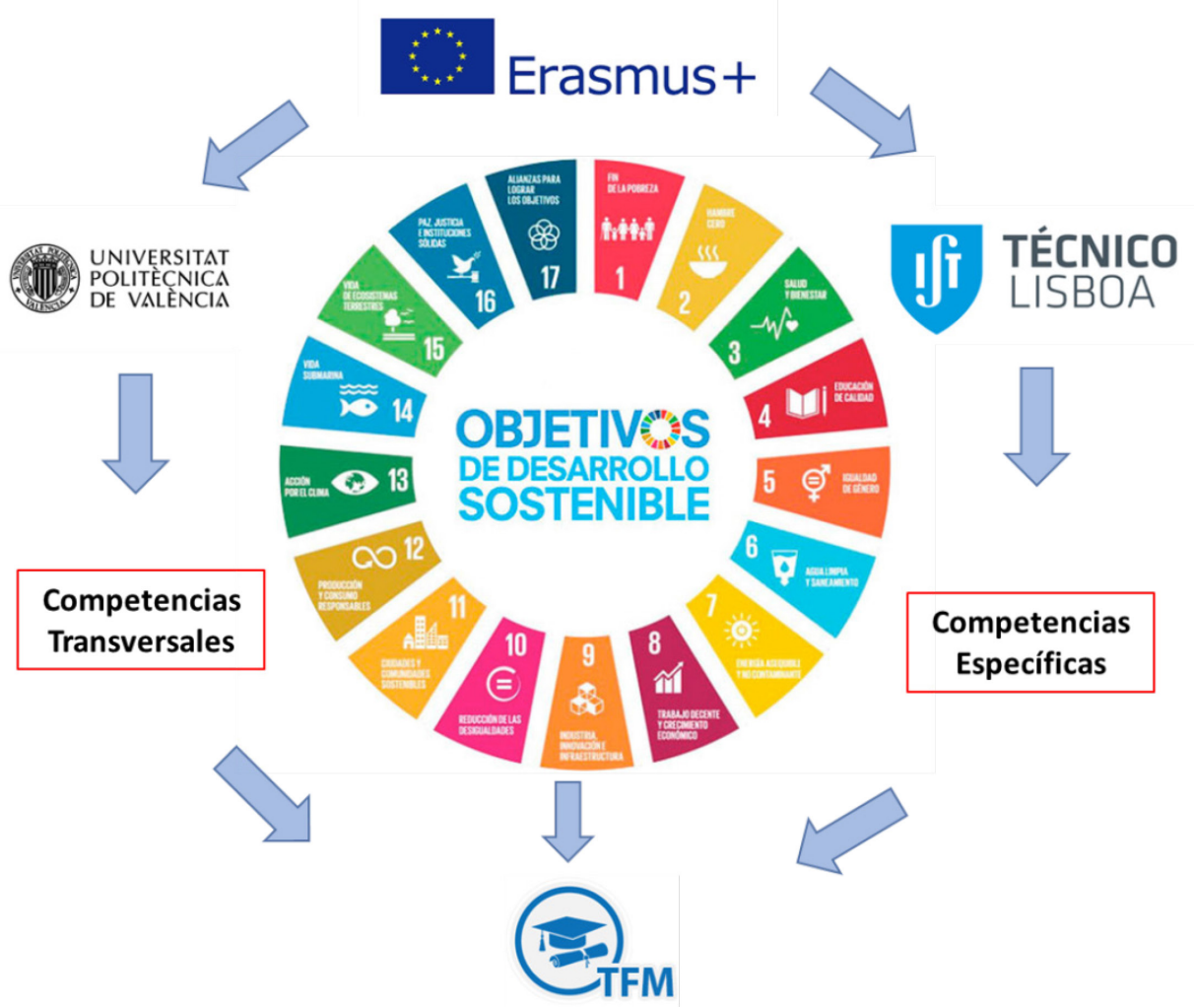

Fig 1. Relación de competencias, ODS y desarrollo de TFG y TFM

\section{Desarrollo de la innovación docente}

El desarrollo de un TFG o TFM supone por parte del alumno un esfuerzo de integrar las competencias específicas que ha desarrollado durante el título, así como poner en práctica parte, sino todas de las competencias transversales que desde el curso 2015 fueron implantadas en el plan UPV 2015-2020 (UPV, 2015). Entre estas competencias, en el desarrollo de un TFG y/o TFM de una titulación de ingeniería, destaca el uso de las siguientes competencias: aprendizaje permanente, pensamiento crítico, análisis y resolución de problemas, responsabilidad ética, profesional y medio ambiental, diseño y proyecto e instrumental específica, siendo la competencia diseño y proyecto, fundamental en el desarrollo de un TFG y/o TFM.

Desde el grupo de trabajo planteado, se han propuesto diferentes temas en los que el alumno o alumna no tenga solo que poner en práctica las competencias específicas del título y las transversales, sino que deban poner en valor algunos de los ODS planteados en la Agenda 2030, para dotar al TFM y/o TFG de un valor social de transferencia del conocimiento. Esta "transferencia" puede ser el punto de partida para el desarrollo de anteproyectos y estudios de viabilidad para poner en marcha dichos proyectos en zonas en desarrollo.

La coordinación de los TFM y TFG es planteada con una coordinación no presencial entre los grupos de profesores del PDI de la UPV e IST. Además, la coordinación está apoyada con dos reuniones anuales presenciales (una en Lisboa (Portugal) y otra en Alcoy o Valencia (España)) mediante el uso de las ayudas propuestas por el plan ERASMUS+STA al profesorado. 


\section{Planificación del desarrollo del TFM y/o TFG}

En todos los casos, el alumno cuenta con una rúbrica, similar a la desarrollada por (Pérez-Sánchez et al., 2018), en la que desarrolla los diferentes indicadores aplicados al TFG y/o TFM, desarrollando indicadores específicos asociados al TFG/TFM. La Tabla 1 muestra un ejemplo aplicado a TFG. Los indicadores analizados y/o evaluados, que cada uno de ellos es desarrollado en indicadores específicos acorde al TFG/TFM son: (1) Fundamentar el contexto y la necesidad del proyecto; (2) Formular los objetivos generales y específicos del proyecto; (3) Planificar las acciones para desarrollar la metodología y cumplir los objetivos; (4) Identificar los posibles riesgos y viabilidad del proyecto; (5) Revisión y análisis de los resultados.

Tabla 1. Ejemplo de rúbrica propuesta para el desarrollo de un TFG relacionado en el área de ingeniería hidráulica (Pérez-Sánchez et al., 2018)

\begin{tabular}{|c|c|c|c|c|}
\hline \multirow{2}{*}{ INDICADORES } & \multicolumn{4}{|c|}{ DESCRIPTORES } \\
\hline & D. No alcanzado & C. En desarrollo & B. Bien /adecuado & A. Excelente/ejemplar \\
\hline $\begin{array}{l}\text { 1. Fundamenta el } \\
\text { contexto y la } \\
\text { necesidad del } \\
\text { proyecto }\end{array}$ & $\begin{array}{l}\text { Alude a la necesidad } \\
\text { del proyecto, pero no } \\
\text { la argumenta }\end{array}$ & $\begin{array}{l}\text { Justifica la necesidad del } \\
\text { proyecto en base a } \\
\text { opiniones y juicios } \\
\text { escasamente contrastados } \\
\text { y fundamentados }\end{array}$ & $\begin{array}{l}\text { Fundamenta el contexto y } \\
\text { necesidad del proyecto } \\
\text { correctamente de forma } \\
\text { correcta pero no completa }\end{array}$ & $\begin{array}{c}\text { Fundamenta el contexto y } \\
\text { necesidad del proyecto correcta y } \\
\text { completamente }\end{array}$ \\
\hline $\begin{array}{l}\text { Introducción, } \\
\text { objetivos y } \\
\text { justificación }\end{array}$ & $\begin{array}{l}\text { No existe el primer } \\
\text { punto }\end{array}$ & $\begin{array}{l}\text { Introduce el proyecto a } \\
\text { realizar, pero no justifica } \\
\text { su necesidad (o lo hace } \\
\text { atendiendo a criterios } \\
\text { equivocados) }\end{array}$ & $\begin{array}{c}\text { Introduce el proyecto, define } \\
\text { objetivos, pero la } \\
\text { justificación no atiende a } \\
\text { necesidades concretas }\end{array}$ & $\begin{array}{c}\text { Introduce el proyecto, define } \\
\text { claramente los objetivos y lo } \\
\text { justifica aludiendo a necesidades } \\
\text { concretas. }\end{array}$ \\
\hline $\begin{array}{c}\text { 2. Formula los } \\
\text { objetivos del } \\
\text { proyecto con } \\
\text { coherencia respecto a } \\
\text { las necesidades } \\
\text { detectadas en el } \\
\text { contexto } \\
\end{array}$ & $\begin{array}{l}\text { Formula objetivos sin } \\
\text { aludir a necesidades }\end{array}$ & $\begin{array}{c}\text { Formula objetivos no } \\
\text { coherentes con las } \\
\text { necesidades }\end{array}$ & $\begin{array}{c}\text { Formula objetivos coherentes } \\
\text { con las necesidades }\end{array}$ & $\begin{array}{l}\text { Formula objetivos coherentes con } \\
\text { las necesidades y, además, son } \\
\text { adecuados y originales para la } \\
\text { transformación de la situación } \\
\text { actual atendiendo las necesidades } \\
\text { detectadas }\end{array}$ \\
\hline $\begin{array}{l}\text { Introducción, } \\
\text { objetivos y } \\
\text { justificación }\end{array}$ & $\begin{array}{l}\text { No existe el primer } \\
\text { punto }\end{array}$ & $\begin{array}{c}\text { Establece objetivos, pero } \\
\text { son ambiguos o imprecisos }\end{array}$ & $\begin{array}{c}\text { Establece objetivos de forma } \\
\text { suficiente }\end{array}$ & $\begin{array}{c}\text { Establece objetivos claros y } \\
\text { operativos }\end{array}$ \\
\hline $\begin{array}{l}\text { 3. Planifica las } \\
\text { acciones con eficacia } \\
\text { (logra objetivos) }\end{array}$ & $\begin{array}{l}\text { Planifica acciones no } \\
\text { eficaces (no logra } \\
\text { gran parte de los } \\
\text { objetivos) }\end{array}$ & $\begin{array}{l}\text { Planifica acciones } \\
\text { parcialmente eficaces } \\
\text { (logra parte de los } \\
\text { objetivos) }\end{array}$ & $\begin{array}{c}\text { Planifica acciones eficaces en } \\
\text { su gran mayoría (logra gran } \\
\text { parte de los objetivos) }\end{array}$ & $\begin{array}{c}\text { Planifica acciones completamente } \\
\text { eficaces (todos los objetivos han } \\
\text { sido cumplidos) }\end{array}$ \\
\hline $\begin{array}{c}\text { Para cada uno de los } \\
\text { puntos }\end{array}$ & $\begin{array}{l}\text { No existe ningún tipo } \\
\text { de introducción del } \\
\text { punto a desarrollar }\end{array}$ & $\begin{array}{c}\text { Hace una breve } \\
\text { descripción de lo contiene } \\
\text { el punto }\end{array}$ & $\begin{array}{c}\text { Describe y justifica el punto } \\
\text { a desarrollar, pero desde una } \\
\text { perspectiva académica }\end{array}$ & $\begin{array}{c}\text { Describe y justifica la necesidad } \\
\text { del punto a desarrollar desde un } \\
\text { punto de vista técnico }\end{array}$ \\
\hline $\begin{array}{l}\text { 4. Planifica las } \\
\text { acciones con } \\
\text { eficiencia (usa los } \\
\text { recursos de forma } \\
\text { óptima) } \\
\end{array}$ & $\begin{array}{c}\text { No planifica acciones } \\
\text { eficientes }\end{array}$ & $\begin{array}{c}\text { Planifica acciones } \\
\text { eficientes, aunque } \\
\text { visiblemente mejorables }\end{array}$ & $\begin{array}{l}\text { Planifica acciones eficientes, } \\
\text { pero no todas }\end{array}$ & $\begin{array}{l}\text { Planifica de forma completa } \\
\text { acciones eficientes }\end{array}$ \\
\hline Curva de consigna & $\begin{array}{l}\text { No se realiza el } \\
\text { cálculo }\end{array}$ & $\begin{array}{l}\text { Se realiza, pero es } \\
\quad \text { incorrecto }\end{array}$ & $\begin{array}{c}\text { El resultado es correcto, pero } \\
\text { no realiza una discusión del } \\
\text { mismo }\end{array}$ & $\begin{array}{c}\text { El resultado es correcto y el } \\
\text { alumno desarrolla un análisis de } \\
\text { los resultados. }\end{array}$ \\
\hline $\begin{array}{c}\text { Definición del } \\
\text { volumen del depósito }\end{array}$ & $\begin{array}{l}\text { No se realiza el } \\
\text { cálculo }\end{array}$ & $\begin{array}{l}\text { Se realiza, pero es } \\
\text { incorrecto }\end{array}$ & $\begin{array}{c}\text { El resultado es correcto, pero } \\
\text { no realiza una discusión del } \\
\text { mismo }\end{array}$ & $\begin{array}{c}\text { El resultado es correcto y el } \\
\text { alumno desarrolla un análisis de } \\
\text { los resultados. }\end{array}$ \\
\hline $\begin{array}{l}\text { Selección de las } \\
\text { bombas }\end{array}$ & $\begin{array}{l}\text { No se realiza la } \\
\text { selección }\end{array}$ & $\begin{array}{l}\text { Se realiza, pero es } \\
\quad \text { incorrecta }\end{array}$ & $\begin{array}{c}\text { El resultado es correcto, pero } \\
\text { no realiza una discusión del } \\
\text { mismo }\end{array}$ & $\begin{array}{c}\text { El resultado es correcto y el } \\
\text { alumno desarrolla un análisis de } \\
\text { los resultados proponiendo otros } \\
\text { modelos o soluciones }\end{array}$ \\
\hline
\end{tabular}




\section{Resultados}

\section{Titulaciones afectadas}

Desde el grupo de profesores, las titulaciones implicadas son:

1) Master en Ingeniería Electrónica y de Computadores. Titulación impartida en el IST de Lisboa. En este caso, son estudiantes de máster entre 21 y 25 años, que previamente han cursado un grado y se encuentran actualmente en su fase final del máster, con pocas asignaturas pendientes o únicamente con el TFM para concluir sus estudios. Estos estudiantes carecen de competencias específicas de ingeniería hidráulica y de máquinas hidráulicas, a excepción de la asignatura troncal de mecánica de fluidos que recibieron en sus estudios de grado. Por tanto, los conocimientos necesarios en términos hidráulicos son adquiridos mediante el uso de recursos online, principalmente polimedias (López-Jiménez y Pérez-Sánchez., 2016) así como los seminarios impartidos en las diferentes estancias ERASMUS+ STA (López-Jiménez y PérezSánchez., 2019)

2) Máster Universitario en Ingeniería Hidráulica y Medio Ambiente. Titulación impartida en el Campus de Vera de la UPV. Al igual que en el caso anterior, son alumnos y alumnas en la etapa final en los estudios del máster entre 21 y 25 años. En este caso, las competencias específicas adquiridas están fuertemente relacionadas con la ingeniería hidráulica y los estudiantes únicamente han cursado de manera generalistas los conocimientos en el área de ingeniería electrónica. Es, a través de seminarios y docencia online, como los estudiantes complementan los conocimientos necesarios para el análisis hidroeléctrico de los sistemas.

3) Grado de Ingeniería Mecánica. Titulación impartida en el Campus de Alcoy de la UPV. Los alumnos que cursan estos estudios tienen una edad comprendida entre los 18 y 23 años mayoritariamente. Cuando el alumno o alumna está realizando el TFG, generalmente ha cursado la mayoría de las asignaturas de cuarto curso, quedando únicamente el TFG o créditos residuales del último curso. Dentro de este grupo de estudiantes, necesitan una formación complementaria en conceptos de electricidad, y en ocasiones, si el TFG está fuerte relacionado con un tema en concreto, deben profundizar en conceptos hidráulicos, mediante la asistencia de seminarios, visionado de polimedias y/o análisis de artículos de investigación publicados.

\section{Casos de estudio I. Trabajos Finales de Master}

Dos trabajos finales de master se engloban dentro de este primer caso de estudio. Concretamente, el caso de estudio está aplicado a un sistema de regadío "Macuvulane 1". Esta explotación agrícola está ubicada en el distrito de Magude (Mozambique, Figura 2a) en las coordenadas ETRS89 25\%01'33.9"S y $32^{\circ} 40^{\prime} 49.4$ "E. (Figura 2b).

1) Uso de paneles fotovoltaicos con almacenamiento de energía hidroeléctrica bombeada para el sistema de riego de la plantación de caña de azúcar en el distrito de Magude-Mozambique

El estudio de caso se centra en 200 hectáreas de riego por aspersión para caña de azúcar en Mozambique. El área irrigada es explotada por una asociación campesina de Xinavane, compuesta por aproximadamente doscientas personas a. La única fuente de agua es el río Incomati, ubicado en el punto de suministro con el riego a unos $5 \mathrm{~km}$ de distancia. Asimismo, el relieve presenta una pendiente variable con pendientes que alcanzan el $10 \%$. Actualmente, la asociación es responsable de todo el proceso de producción, incluida la gestión del agua y la energía. 
En este contexto, la implementación de mecanismos de gestión de energía innovadores y eficientes es de suma importancia para el éxito de la producción. La sostenibilidad del riego es un desafío, dados los costes asociados con la electricidad que son respaldados por las asociaciones campesinas. Por lo tanto, se necesita un estudio para reemplazar la fuente de energía para alimentar las bombas eléctricas y, en un primer enfoque, utilizar la energía fotovoltaica con almacenamiento de energía, aprovechando también el flujo del río para la producción de energía eléctrica utilizando un generador de inducción.

2) Uso de paneles fotovoltaicos con almacenamiento de energía hidroeléctrica bombeada para el sistema de riego de la plantación de caña de azúcar en el distrito de Magude-Mozambique

La tesis de máster se centra en proponer una metodología de optimización analítica que permita el mayor grado posible de suficiencia eléctrica a bajo costo utilizando paneles fotovoltaicos con almacenamiento de energía hidroeléctrica bombeada para un sistema de riego de la plantación de caña de azúcar se encuentra en el distrito de Magude (Mozambique). La sostenibilidad del riego es un desafío, dados los costos asociados con la electricidad que son respaldados por las asociaciones campesinas. Por lo tanto, se necesita un estudio para reemplazar la fuente de energía para alimentar las bombas eléctricas y, en un primer enfoque, utilizar la energía fotovoltaica con almacenamiento de energía, aprovechando el flujo del río para el establecimiento de depósitos que actúan como almacenes de energía potencial para períodos en los que la energía renovable es excedente.

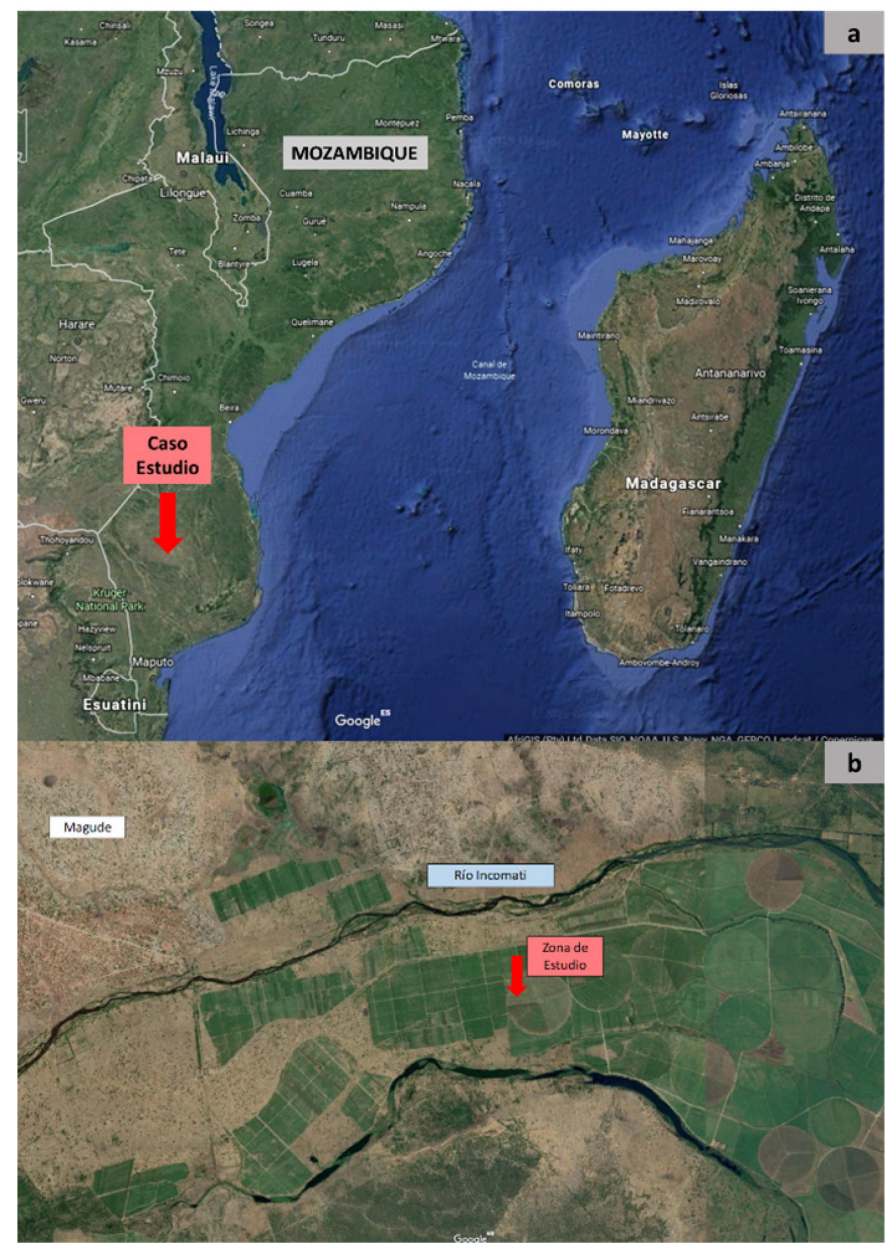

Fig 2. Caso de estudio aplicado a los TFM 


\section{Casos de estudio II. Trabajo Final de Grado}

1) Diseño y Estudio Técnico de una instalación hidroeléctrica para microgeneración en Buenos Aires (Argentina).

En este caso, se desarrolla un trabajo final de grado tiene por objetivo el diseño y estudio técnico de una instalación hidroeléctrica destinada al autoconsumo en la región de Buenos Aires, Argentina. Debido a la problemática ocasionada por los constantes cortes en el suministro eléctrico que ocurren en este país, surge la necesidad de buscar soluciones alternativas para solventar esta carencia, razón fundamental para el desarrollo de este proyecto. La instalación hidroeléctrica que será diseñada consta de un circuito cerrado compuesto por una bomba centrífuga, la cual alimenta a una turbina Pelton de dos inyectores que se encuentra conectada a un generador, el cual es utilizado para acumular energía eléctrica en baterías mientras existe suministro eléctrico nacional, para luego utilizarla cuando haya cortes de dicho suministro. La turbina descarga en un estanque, el cual se encuentra conectado a la bomba cerrando así el sistema de generación. El proyecto será realizado tomando como datos de partida la geometría de una rueda Pelton de la que se dispone, determinando a partir de estos el resto de los componentes de la instalación.

\section{Conclusiones}

La integración de los ODS dentro de los TFG y TFM dirigidos ha supuesto que los estudiantes pudieron desarrollar un cierto grado de pensamiento crítico, imaginativo e innovador sobre el desarrollo sostenible en general y los ODS en particular. Este hecho, ha permitido que los alumnos puedan interrelacionar las competencias básicas y específicas de la titulación y las competencias transversales, transfiriendo ese conocimiento a la mejora de los ODS dentro de zonas desfavorecidas que mejoren la calidad de vida de las personas.

El proyecto llevado a cabo por los profesores del DIHMA-UPV y del DIEC-IST ha supuesto un reto personal desde el punto de vista de la integración de conocimientos que el alumno había adquirido de forma básica, puesto que sus titulaciones estaban enfocadas en ingeniería electrónica (como es el caso de los estudiantes del Máster de Ingeniería Electrónica que cursan en el IST) o hidráulica (como es el caso de los estudiantes del Grado de Ingeniería Mecánica o el Máster de Ingeniería Hidráulica y Medio Ambiente). El uso de herramientas asíncronas para mejorar los resultados de aprendizaje en estos alumnos en el área de hidráulica y electricidad han supuesto un aspecto clave en la mejora del desarrollo de los TFG y TFM.

El desarrollo de estas actividades se ha llevado a cabo gracias al uso de las ayudas Erasmus+STA que ha permitido que los profesores de ambas universidades puedan visitar una vez al año los campus del país vecino, dando lugar a la mejora de la coordinación y desarrollo de los trabajos que el resto del año se llevan a cabo mediante webminars.

La satisfacción del alumno que desarrolla un TFG o TFM aplicado a una zona desfavorecida mejorando la calidad de vida de las personas se ve incrementada frente a un TFG o TFM clásico. Esta impresión es obtenida a través de la observación del estudiante, ya que no se han realizado ningún tipo de encuesta, pero sí que se deduce de la observación del alumno por parte de los profesores, comparándolo con otros alumnos que desarrollaron TFG/TFM "clásicos". Esto destaca la sensibilidad que el estudiante muestra por este aspecto dentro de su responsabilidad ética, medioambiental y profesional.

Hasta la fecha se han desarrollado 4 TFM los cuales se encuentran ya defendidos, estando actualmente 3 TFM en redacción y 1 TFG.

(cc)) BY-NC-ND 2020, Universitat Politècnica de València

Congreso In-Red (2020) 


\section{Agradecimientos}

La experiencia aquí descrita se ha llevado a cabo como parte de los trabajos desarrollados en el EICE DESMAHIA "Desarrollo de metodologías activas y estrategias de evaluación aplicadas al campo de la Ingeniería Hidráulica". Los autores agradecen a la UPV, y en particular al ICE, el apoyo prestado para llevar a cabo esta iniciativa.

\section{Referencias}

BANCO MuNDIAL (2019). https://datos.bancomundial.org/indicador/EG.ELC.ACCS.ZS [Consultado 20/02/2020]

DESA, U. N. (2016). Transforming our world: The 2030 agenda for sustainable development.

LÓPEZ JimÉnEZ, PA; PÉREZ-SÁnChEZ, M (2016). Los objetos de aprendizaje como conductores para favorecer el aprendizaje autónomo en el campo de la mecánica de fluidos.. EN Congreso Nacional de Innovación Educativa y Docencia en Red = Congrés Nacional d'Innovació Educativa i Docència en Xarxa (IN-RED 2016). (53 - 60). Valencia, España: Editorial Universitat Politècnica de València.

LÓPEZ JiMÉNEZ, PA; PÉREZ-SÁNCHEZ, M (2019). Los seminarios apoyados con formación online como estrategia de aprendizaje autónomo en últimos cursos de Máster: caso práctico en el MUIHMA-UPV. EN V Congreso Nacional de Innovación Educativa y Docencia en Red (IN-RED 2019). (459 - 466). Valencia, Spain: Editorial Universitat Politècnica de València.

KopninA, H. (2018). Teaching sustainable development goals in The Netherlands: a critical approach. Environmental education research, 24(9), 1268-1283.

OdS (2019). Organización Mundial de las Naciones Unidas. Objetivos de Desarrollo Sostenible. https://www.un.org/sustainabledevelopment/es/energy/ [Consultado 20/08/2020]

Pérez-Sánchez, M., Vinaches Ramis, J., Satorre-Aznar, JR., LóPez Jiménez, PA (2018). “CT-05. Diseño y Proyecto" integrada en la evaluación la asignatura Ingeniería Fluidomecánica, asociada al PIME B24. EN IV Congreso Nacional de Innovación Educativa y Docencia en Red (IN-RED 2018). Valencia, España: Editorial Universitat Politècnica de València.

UPV (2015). Universitat Politècnica de València. Institutional project of the generic outcomes. https://www.upv.es/entidades/ICE/info/Proyecto_Institucional_CT.pdf [05/03/2020] 